\title{
Role of tropomyosin in silkworm allergy
}

\author{
KYOUNG YONG JEONG ${ }^{1}$, IN-SOO HAN ${ }^{1}$, JUNE YONG LEE ${ }^{1,2}$, \\ KYUNG HEE PARK ${ }^{1}$, JAE-HYUN LEE ${ }^{1}$ and JUNG-WON PARK ${ }^{1}$ \\ ${ }^{1}$ Department of Internal Medicine, Institute of Allergy; ${ }^{2}$ Brain Korea 21 PLUS Project for Medical Science, \\ Yonsei University College of Medicine, Seoul 03722, Republic of Korea
}

Received January 27, 2016; Accepted January 27, 2017

DOI: $10.3892 / \mathrm{mmr} .2017 .6373$

\begin{abstract}
Silkworm pupae are widely consumed in Asian countries and allergic reactions following consumption have been described. However, false-positive responses in skin prick allergy tests or non-specific immunoglobulin $\mathrm{E}(\mathrm{IgE})$ responses to total extract of silkworm pupa make diagnosis difficult. Although improved allergy diagnosis is required, molecular characterization of silkworm allergens has not been performed to date, except for Bomb $\mathrm{m}$ 1, an arginine kinase. This study aimed to evaluate the allergenicity of tropomyosin, a well-established invertebrate pan-allergen, from silkworm pupa. The silkworm tropomyosin gene was cloned by reverse transcription and polymerase chain reaction, and the protein was overexpressed in Escherichia coli and purified by affinity chromatography using Nickel-resin. IgE reactivity of the recombinant protein was examined by ELISA and competitive inhibition analyses. Silkworm pupa tropomyosin shared 73.5-92.3\% amino acid sequence identity with previously identified allergenic tropomyosins. Sera from eight of 15 patients with silkworm allergy (53.3\%) exhibited binding of IgE to the recombinant protein. However, recombinant protein was able to inhibit less than $10 \%$ of $\operatorname{IgE}$ reactivity to silkworm pupa extract. Of the eight sera tested, six that specifically reacted with silkworm tropomyosin also demonstrated $\operatorname{IgE}$ reactivity to shrimp and crab. In the present study, specific IgE to silkworm tropomyosin was detected in patients with silkworm allergy, suggesting that it may be useful in diagnosis of allergy to silkworm pupa.
\end{abstract}

\section{Introduction}

Traditional use of insects as food is widespread in tropical and subtropical countries because it provides nutritional and

Correspondence to: Professor Jung-Won Park or Dr Kyoung Yong Jeong, Department of Internal Medicine, Institute of Allergy, Yonsei University College of Medicine, 50-1 Yonsei Street, Seoul 03722, Republic of Korea

E-mail: parkjw@yuhs.ac

E-mail: jeongky@yuhs.ac

Key words: allergen, silkworm, tropomyosin economic benefits in rural communities (1). Certain insects, including silkworms, have also been used as therapeutics in Korean traditional medicine (2). Because of its high nutritional value, the silkworm has also been considered as a potential solution to the exhaustion of food sources (3). Silkworm pupa (SWP) is a traditional food that is commonly consumed following boiling with soybean sauce. In China, oil-fried, water-boiled or ground pupa is often consumed. It has been reported that $>1,000$ patients per year suffer anaphylactic reactions following consumption of SWP in China (4). A foreign tourist was reported to enter anaphylactic shock after consuming SWP (4). However, a high prevalence of skin test reactivity (9.4\%) to total extract of SWP among patients with allergies unrelated to SWP was reported, suggesting that non-specific or clinically irrelevant immunoglobulin (Ig) E binding is a substantial problem in the diagnosis of SWP allergy (5).

A retrospective analysis of serum $\mathrm{IgE}$ to silkworm moth and 9 common inhalant allergens among patients with respiratory allergy in Guangzhou, China, demonstrated a positive correlation between allergies to silkworm moths and to cockroaches or house dust mites. Cross-allergenicity between moth and midge was also described in Japanese asthmatic patients (6). Baldo and Panzani (7) described IgE-binding components of $\sim 37 \mathrm{kDa}$, potentially tropomyosin, from various arthropods, including house fly, blowfly, common clothes moth, warehouse moth, Bogong moth, grain borer, locust, silverfish, cockroach, carpet beetle and house dust mite (7). However, the IgE reactivity of silkworm tropomyosin has not been investigated.

It is well established that the pan-allergen tropomyosin, especially from invertebrates including shellfish and mollusks, is an important cause of food allergy (8). Tropomyosin from dust mites, cockroaches, and helminthes elicits allergic symptoms and it is conceivable that tropomyosin is important in SWP allergy, especially considering its heat stable nature. In the present study, recombinant SWP tropomyosin was produced and its IgE reactivity was evaluated for diagnosis of SWP allergy.

\section{Materials and methods}

SWP protein extract preparation. A frozen SWP was provided by Anysilk Co., Ltd. (Cheongju, Korea). Following pulverization in liquid nitrogen, the pupa was defatted with five volumes of a 1:1 mixture of ethyl ether and ethyl acetate. Proteins 
were extracted with phosphate-buffered saline (PBS; pH 7.4) containing $6 \mathrm{mM} 2$-mercaptoethanol, protease inhibitor cocktail set III (1:1,000; Calbiochem; Merck KGaA, Darmstadt, Germany), and $1 \mathrm{mg} / \mathrm{ml}$ 1-phenyl-3-(2-thiazyl)-2-thiourea (Sigma-Aldrich; Merck KGaA) at $4^{\circ} \mathrm{C}$. The supernatant was collected after centrifugation at $10,000 \times \mathrm{g}$ for $30 \mathrm{~min}$ at $4^{\circ} \mathrm{C}$, filtered through a syringe $(0.22 \mu \mathrm{m}$; Merck KGaA $)$, and stored at $-70^{\circ} \mathrm{C}$ until use. The protein concentration was determined by Bradford assay (Bio-Rad Laboratories, Inc., Hercules, CA, USA).

Subjects and serum samples. Serum samples were obtained from 15 patients (age range, 17-43 years; mean, 34) who visited the Allergy-Asthma Center at Severance Hospital (Seoul, Korea) between 2014 and 2015. The patients were diagnosed with SWP allergy based on the temporal relationship between SWP intake and onset of cutaneous allergic symptoms and a positive skin prick test to total extract of SWP (Table I). These symptoms included urticaria and anaphylaxis, and occurred within 30 min following intake. ImmunoCAP (Thermo Fisher Scientific, Inc., Waltham, MA, USA) assay was performed for common allergens, including shrimp (f24) and crab (f23), in order to estimate potential cross-reactivity. ImmunoCAP was not performed for SWP because it is not commercially available. Serum samples from 9 individuals (age range, 3-47 years; mean, 21) who were not allergic to SWP and exhibited no positive reaction to the allergen by skin prick test and ImmunoCAP were also included (healthy controls). The present study was approved by the relevant institutional review board at the Yonsei University College of Medicine (4-2013-0397).

Multiple sequence alignment of silkworm tropomyosin with allergenic species. Analysis of silkworm tropomyosin was performed using the Basic Local Alignment Search Tool (BLAST) software from the National Center for Biotechnology Information (NCBI; Bethesda, MD, USA) (9). A homology search in GenBank was performed using the NCBI BlastX program. Sequence alignment with the retrieved allergen sequences was performed using the CLUSTAL $X$ software (10).

Production of recombinant tropomyosin. Total RNA was prepared from frozen pupae, which were provided by Anysilk Co., Ltd., with TRIzol reagent (Invitrogen; Thermo Fisher Scientific, Inc.). First strand cDNA was synthesized from $5 \mu \mathrm{g}$ of total RNA using AMV reverse transcriptase (Promega Corporation, Madison, WI, USA). The reaction mixture contained $0.5 \mu \mathrm{M}$ oligo-dT $\left(\mathrm{T}_{18}\right)$ primer (Promega Corporation), 1.9 U of RNase inhibitor (RNasin ${ }^{\circledR}$; Promega Corporation), 1x reaction buffer (Promega Corporation), $0.5 \mathrm{mM}$ dNTPs (Promega Corporation), $4 \mathrm{mM}$ sodium pyrophosphate (Sigma-Aldrich; Merck KGaA) and $5 \mathrm{U}$ of reverse transcriptase, reverse transcription was performed at $42^{\circ} \mathrm{C}$ for $1 \mathrm{~h}$. Polymerase chain reaction (RT-PCR) was performed using oligonucleotide primers designed based on the tropomyosin GenBank sequence (accession no. NM_001110312), as follows: Forward, 5'-ATGGACGCGATCAAGAAGAA-3'; and reverse, 5'-TTATTCCTTGAGGATGAGCT-3'. After 5 min of initial denaturation at $95^{\circ} \mathrm{C}, 35$ cycles of $\mathrm{PCR}$ reaction $(5 \mathrm{sec}$ at $94^{\circ} \mathrm{C}, 5 \mathrm{sec}$ at $50^{\circ} \mathrm{C}$ and $10 \mathrm{sec}$ at $72^{\circ} \mathrm{C}$ ) were performed with
HiPi Super DNA polymerase (HiPi Super 5x PCR Master Mix; ELPIS-Biotech, Inc., Daejeon, Korea). The PCR-amplified DNA fragment, without purification, was directly ligated into pEXP-5NT/TOPO vector (Invitrogen). Following transformation of Escherichia coli BL21 (DE3), transformants were cultured at $37^{\circ} \mathrm{C}$ in $1 \mathrm{~L}$ of Luria Bertani broth and expression of recombinant protein was induced by the addition of $0.8 \mathrm{mM}$ isopropyl-1-thio- $\beta$-D-galactopyranoside when absorbance at $600 \mathrm{~nm}$ was 0.5 . The culture was harvested $4 \mathrm{~h}$ after induction. The cellular pellets were resuspended in buffer $(10 \mathrm{mM}$ imidazole, $300 \mathrm{mM} \mathrm{NaCl}, 50 \mathrm{mM} \mathrm{NaH}_{2} \mathrm{PO}_{4}, \mathrm{pH} 8.0$ ), and lysed by sonication. The lysates were centrifuged at $10,000 \times \mathrm{g}$ at $4^{\circ} \mathrm{C}$ for $30 \mathrm{~min}$, and the recombinant protein was purified from the supernatant (soluble fraction) using nickel-nitrilotriacetic acid agarose (Qiagen GmbH, Hilden, Germany) and analyzed by $10 \%$ SDS-PAGE under reducing conditions. Protein concentration was determined by Bradford assay.

$I g E$ reactivity of recombinant tropomyosin. $\mathrm{IgE}$ reactivity of the recombinant protein was examined by ELISA. A microplate was coated with recombinant protein $(2 \mu \mathrm{g} / \mathrm{ml})$ overnight in $0.05 \mathrm{M}$ sodium bicarbonate buffer ( $\mathrm{pH}$ 9.6). Following blocking with $3 \%$ skim milk in PBS containing $0.05 \%$ Tween-20 (PBST) overnight at $4^{\circ} \mathrm{C}$, serum samples (diluted 1:4) were added and the plate was incubated for $1 \mathrm{~h}$ at room temperature. $\operatorname{IgE}$ antibodies were detected by incubation with biotinylated goat anti-human $\operatorname{IgE}$ (1:1,000; cat. no. BA 3040; Vector Laboratories, Inc., Burlingame, CA, USA) for $1 \mathrm{~h}$, followed by incubation with streptavidin-peroxidase conjugate $(1: 1,000$; Sigma-Aldrich; Merck KGaA) for $30 \mathrm{~min}$. The plate was washed at least three times with PBST between incubation steps. Color development was initiated by addition of the substrate 3,3'5,5'-tetramethyl-benzidine (Kirkegaard \& Perry Laboratories, Inc., Gaithersburg, MD, USA). Absorbance at $450 \mathrm{~nm}$ was measured following addition of $0.5 \mathrm{M} \mathrm{H}_{2} \mathrm{SO}_{4}$ to stop the reaction. The mean absorbance plus 2 standard deviations of the sera from healthy controls was used as a cutoff value for significance.

For ELISA inhibition, microtiter plates were coated with SWP total extract $(10 \mu \mathrm{g} / \mathrm{ml})$ and incubated at $4^{\circ} \mathrm{C}$ overnight. Following blocking with $3 \%$ skim milk in PBST overnight at $4^{\circ} \mathrm{C}$, the wells were incubated for $1 \mathrm{~h}$ at room temperature with patient serum (1:4 dilution, pooled from two patients who showed a positive IgE reaction to the recombinant protein), which had been preincubated for $2 \mathrm{~h}$ at room temperature and overnight at $4^{\circ} \mathrm{C}$ with solutions containing various concentrations of the extract or recombinant proteins. Subsequently, $\operatorname{IgE}$ antibodies were detected as described above. The recombinant 27-kDa hemolymph glycoprotein, a silkworm allergen, was used as an inhibitor for positive control. Production of recombinant $27-\mathrm{kDa}$ glycoprotein was previously described elsewhere (11).

For inhibition of immunoblotting, $10 \mu \mathrm{g}$ of the extract was run on a $12 \%$ SDS-PAGE gel under reducing conditions. The separated proteins were transferred onto polyvinylidene difluoride (PVDF) membrane $(0.45 \mu \mathrm{m}$; Merck KGaA). Following blocking with $3 \%$ skim milk in PBST, the membrane was cut into 4-mm wide strips and incubated overnight with serum sample (1:4 dilution, pooled from two patients who exhibited a positive reaction to recombinant protein), which had been preincubated for $2 \mathrm{~h}$ at room temperature and overnight at $4^{\circ} \mathrm{C}$ with 
Table I. Clinical features of enrolled silkworm allergy patients.

\begin{tabular}{|c|c|c|c|c|c|}
\hline Age & Gender & $\begin{array}{c}\text { SPT wheal to } \\
\text { silkworm pupa }(\mathrm{cm})\end{array}$ & Symptoms & $\begin{array}{l}\operatorname{ImmunoCAP} \\
\left(\mathrm{kU} U_{\mathrm{A}} / \mathrm{l}\right)\end{array}$ & $\begin{array}{c}\text { SPT positive allergens } \\
\text { (degree of skin test response) }\end{array}$ \\
\hline 39 & $\mathbf{F}$ & ND & Anaphylaxis & f23 (1.28), f24 (1.48) & ND \\
\hline 35 & $\mathrm{~F}$ & 3.5 & Urticaria & f23 $(0.68)$, f24 (0.78) & ND \\
\hline 36 & $\mathbf{F}$ & ND & Urticaria & f23 $(0.06)$, f24 $(0.04)$ & ND \\
\hline 37 & $\mathbf{F}$ & 4.5 & Urticaria & f23 (6.55), f24 (6.84) & f4 $(2+)$ \\
\hline 40 & M & ND & Urticaria & $\mathrm{f} 23(0.00), \mathrm{f} 24(0.05)$ & ND \\
\hline 25 & $\mathbf{M}$ & ND & Urticaria & f23 $(0.02)$, f24 (0.05) & ND \\
\hline 43 & M & ND & Anaphylaxis & f23 (11.0), f24 (8.41) & ND \\
\hline 28 & F & 8.5 & Urticaria & $\begin{array}{l}\text { f23 (3.18), f24 (3.64), } \\
\text { f4 (0.02) }\end{array}$ & $\begin{array}{l}\text { Silkworm pupa (3+), } \\
\text { f } 23(1+), \text { f } 24(1+)\end{array}$ \\
\hline 27 & $\mathrm{~F}$ & 4 & Urticaria & $\begin{array}{l}\mathrm{d} 2(1.35), \mathrm{i} 6(0.64), \\
\mathrm{f} 23(0.87), \mathrm{f} 24(1.11)\end{array}$ & $\begin{array}{l}\text { d1 }(2+), \text { i206 }(2+), \\
\text { silkworm pupa }(1+)\end{array}$ \\
\hline 39 & $\mathbf{M}$ & 5.5 & Urticaria & f23 (3.27), f24 (3.27) & Silkworm pupa (-) \\
\hline 18 & $\mathbf{M}$ & 8.5 & Urticaria & f23 (11.3), f24 (10.8) & $\begin{array}{l}\text { Silkworm pupa }(3+), \text { f290 } \\
(2+) \text {, saury }(2+), \text { f206 (1+) }\end{array}$ \\
\hline 30 & M & 2.5 & Anaphylaxis & $\begin{array}{l}\mathrm{f} 23(14.2), \mathrm{f} 24,(11.6), \mathrm{c} 1 \\
(0.15), \mathrm{c} 5(0.35), \mathrm{c} 7(0.28)\end{array}$ & Silkworm pupa $(1+)$ \\
\hline 26 & $\mathbf{M}$ & ND & Urticaria & $\begin{array}{l}\text { d2 (6.34), i6 (3.37), f23 } \\
(5.51), \text { f24 (5.46) }\end{array}$ & d1 $(3+)$, d2 (3+), i206 (2+) \\
\hline 43 & $\mathrm{~F}$ & ND & Urticaria & f23 (0.07), f24 (0.12) & ND \\
\hline 17 & $\mathbf{M}$ & 2 & Urticaria & $\begin{array}{l}\text { d2 (23.0), f23 (8.59), } \\
\text { f24 (8.97) }\end{array}$ & f24 (2.5), silkworm pupa (1+) \\
\hline
\end{tabular}

Patients with positive reaction to the recombinant silkworm tropomyosin are indicated in bold. Degree of skin test response was obtained by comparing the wheal size to histamine control. Each patient was tested for IgE against suspected allergens, which differed between patients. SPT, skin prick test; F, female; M, male; ND, not determined; f23, crab; f24, shrimp; f4, wheat; d2, Dermatophagoides farinae; i6, German cockroach; i206, American cockroach; d1, Dermatophagoides pteronyssinus; f290, oyster; f206, mackerel; c1, Penicilloyl G; c5, Ampicilloyl; c7, Cefaclor.

solutions containing $20 \mu \mathrm{g}$ recombinant protein. Bound $\operatorname{IgE}$ antibodies were detected by incubation with alkaline phosphatase-conjugated goat anti-human IgE (1:1,000; cat. no. A3525; Sigma-Aldrich; Merck KGaA) for $1 \mathrm{~h}$ at room temperature. Color development was initiated by addition of nitro blue tetrazolium (NBT) and 5-bromo-4-chloro-3-indolyl-phosphate (BCIP; Promega Corporation).

Western blot analysis. In order to confirm the presence of tropomyosin in the extract, western blot analysis was performed using the monoclonal antibody, 2G32, raised against German cockroach tropomyosin, Bla g 7 (12). Monoclonal antibody $2 \mathrm{G} 32$ has also been demonstrated to recognize tropomyosin from house dust mite and dusky brown cockroach (13). SWP extract $(10 \mu \mathrm{g})$ was separated on $12 \%$ SDS-PAGE under reducing conditions and Proteins were transferred onto a PVDF membrane. Following blocking overnight with 3\% skim milk in PBST at $4^{\circ} \mathrm{C}$, the membrane was incubated for $1 \mathrm{~h}$ with hybridoma culture supernatant (undiluted, collected after 2-3 days of culture). The blots were then incubated with goat anti-mouse $\mathrm{IgG}$ conjugated with alkaline phosphatase (1:1,000; cat. no. A1293; Sigma-Aldrich; Merck KGaA) for $1 \mathrm{~h}$ at room temperature and developed in a substrate solution of NBT/BCIP.
Statistical analysis. GraphPad Prism 5 (GraphPad Software, Inc., La Jolla, CA, USA) was used for evaluating the statistical parameters. Difference in IgE levels between healthy control and allergic subjects was analyzed with unpaired t-test. The correlation between $\mathrm{IgE}$ to the allergens was evaluated by calculating the Spearman rank correlation coefficient $(\rho)$.

\section{Results}

Homology to allergenic tropomyosins. An alignment analysis was performed on the amino acid sequence of SWP tropomyosin. SWP tropomyosin exhibited up to $92.3 \%$ sequence identity to Chi k 10, a chironomid tropomyosin, followed by $90.1 \%$ to Per a 7 and $89.4 \%$ to Bla g 7 , two cockroach tropomyosins (Fig. 1). SWP tropomyosin also shared 78.5-81.0\% identity with mite tropomyosins (Der p 10, Der f 10, Tyr p 10, and Lep d 10) and 73.5\% identity with shrimp (Pen a 1) and crab (Hom a 1) tropomyosins.

Expression and $I g E$ reactivity of recombinant $S W P$ tropomyosin. The open reading frame of native SWP tropomyosin encodes a 285 -amino acid protein with a calculated molecular mass of $32.8 \mathrm{kDa}$ and an isoelectric point of 4.5 . The coding sequence of SWP tropomyosin was cloned by 


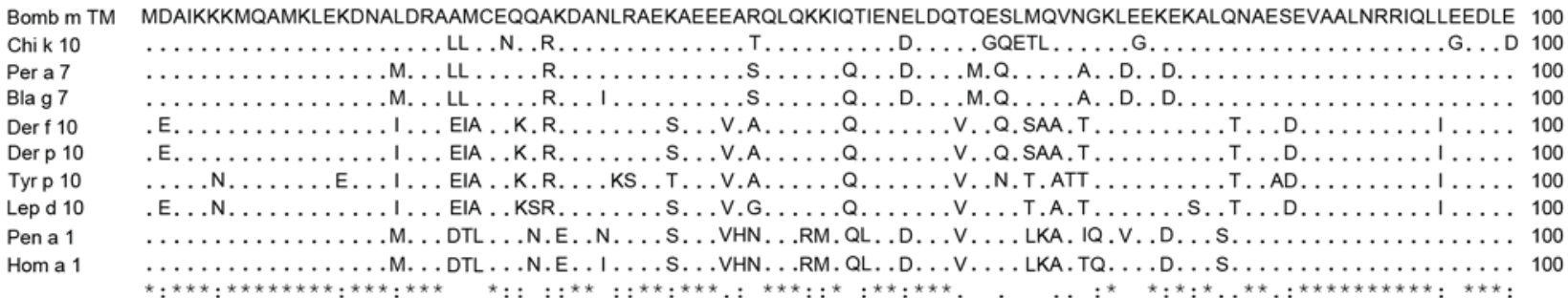

Bomb $m$ TM RSEERLATATAKLSEASQAADESERARKVLENRSLADEERMDALENQLKEARFLAEEADKKYDEVARKLAMVEADLERAEERAESGESKIVELEEELRVV 200

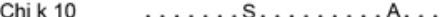

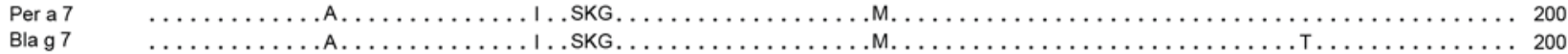

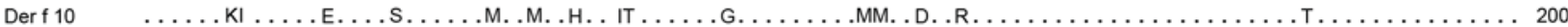

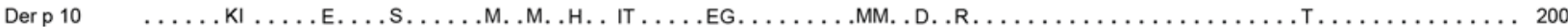

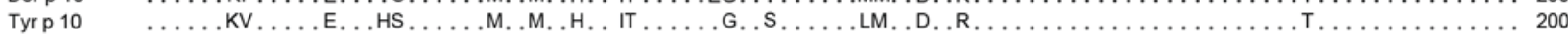

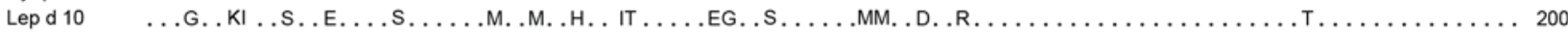

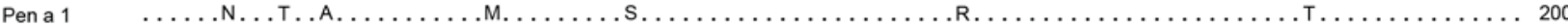

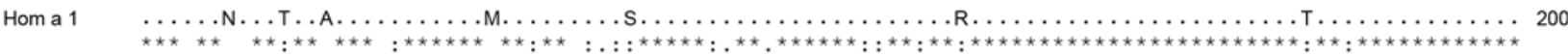

Bomb m TM GNNLKSLEVSEEKANQREEEYKNQIKTLTTRLKEAEARAEFAERSVQKLQKEVDRLEDELVAEKEKYKDIGDDLDTAFVELILKE 285 [285 / 285 , 100.0\%]

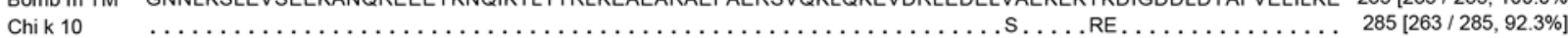

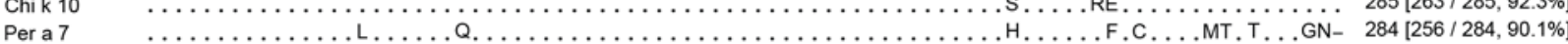

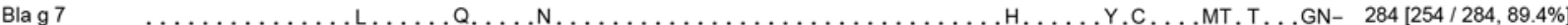

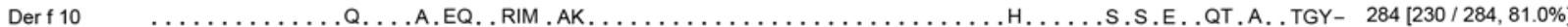

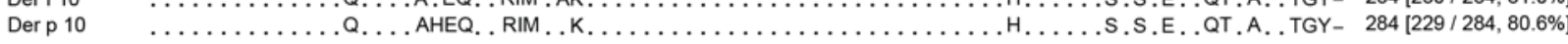

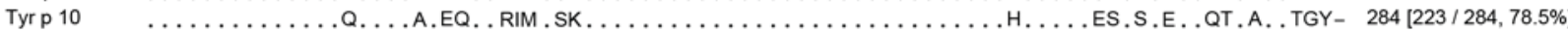

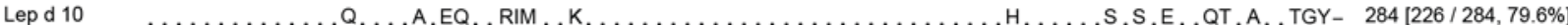

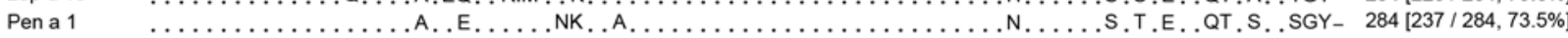

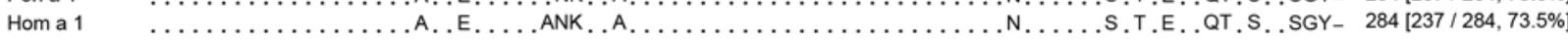

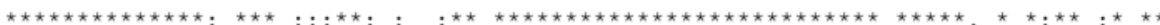

Figure 1. Amino acid sequence alignment of silkworm tropomyosin with other allergenic tropomyosins. The percentage of sequence identity is presented in square brackets. Bomb m TM,_Bombyx mori accession no.NM_001110312; Chi k 10, Chironomus kiiensis accession no.O96764; Per a 7, Periplaneta americana accession no. AF106961; Bla g 7, Blattella germanica accession no. Q9NG56; Der f 10, Dermatophagoides farinae accession no. D17682; Der p 10, Dermatophagoides pteronyssinus accession no. AF016278; Tyr p 10, Tyrophagus putrescenatiae accession no. AY623832; Lep d 10, Lepidoglyphus destructor accession no. AJ250096; Pen a 1, Farfantepenaseus aztecus accession no. DQ151457; Hom a 1, Homarus americanus accession no. AAC48288; *, identical; :, highly conserved; ., less conserved.
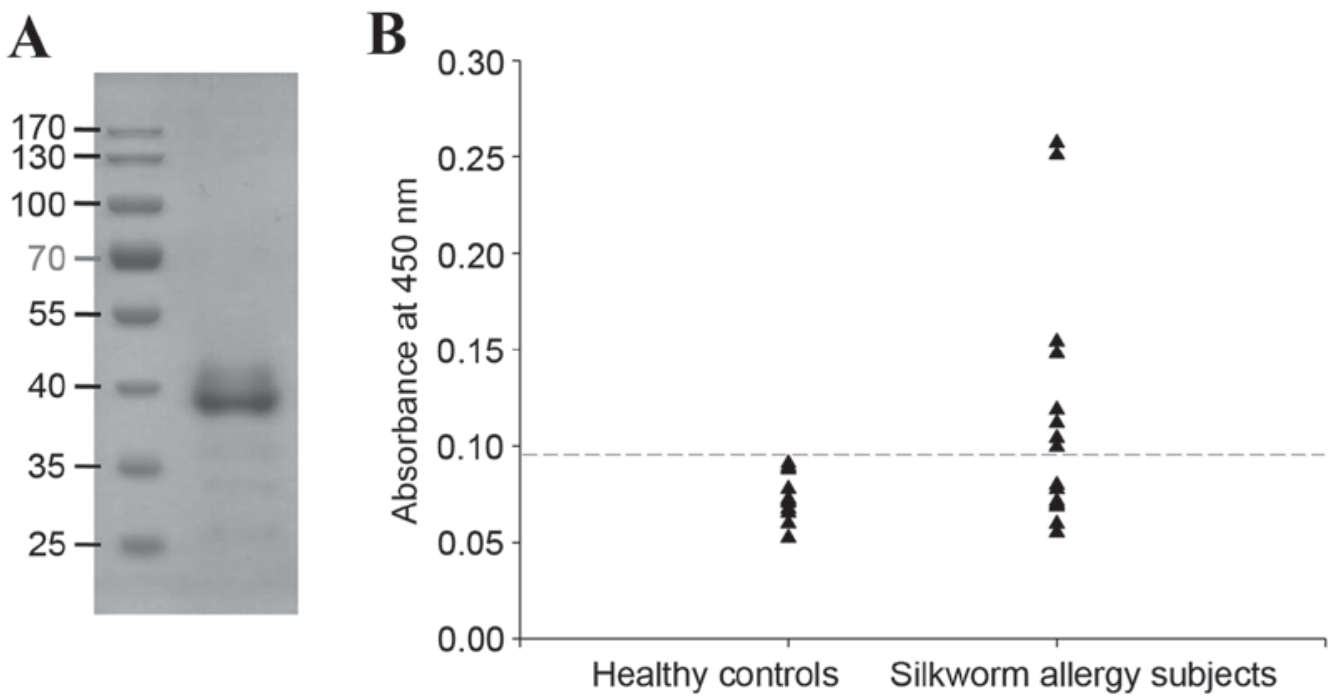

Figure 2. Production of recombinant silkworm tropomyosin and IgE reactivity. (A) Recombinant protein (10 $\mu \mathrm{g})$ was separated in a $12 \%$ polyacrylamide gel containing sodium dodecyl sulfate under reducing conditions and stained with Coomassie Blue. (B) IgE binding of recombinant tropomyosin was tested against sera from patient subjects who were non-allergic (healthy controls) and allergic to silkworm using ELISA; results were expressed as absorbance at $450 \mathrm{~nm}$. Dotted line indicates the cutoff value for significance, which was determined at mean $\operatorname{IgE}$ activity in healthy controls plus $2 \mathrm{x}$ standard variation. $\operatorname{IgE}$, immunoglobulin $\mathrm{E}$.

RT-PCR using specific primers and expressed in E. coli. The resulting purified recombinant tropomyosin migrated as an $\sim 38 \mathrm{kDa}$ protein on SDS-PAGE, even though the molecular mass of recombinant tropomyosin containing the $\mathrm{N}$-terminus
22 amino acid poly-histidine tag (MSGSHHHHHHGSSGE NLYFQSL) was calculated at $35.3 \mathrm{kDa}$ (Fig. 2A). The yield of the purified recombinant tropomyosin was $6.618 \mathrm{mg} / \mathrm{l}$ of bacteria culture. The frequency of $\mathrm{IgE}$ binding to recombinant 
A

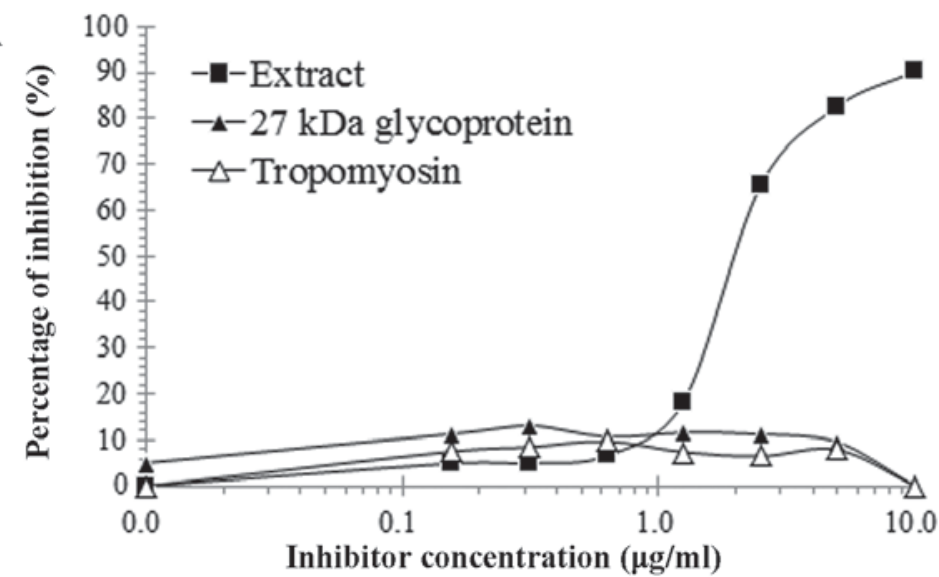

B

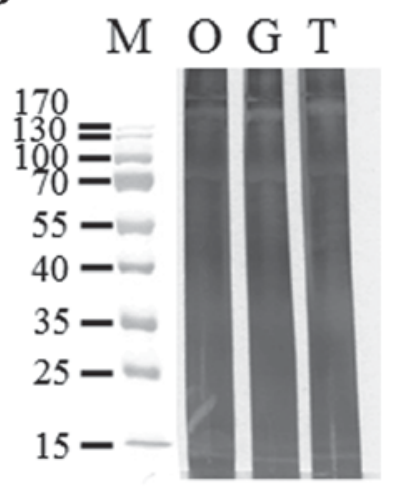

Figure 3. ELISA and immunoblotting inhibition analyses of recombinant proteins. Experiments were performed in duplicate. (A) For inhibition ELISA, IgE binding to silkworm extract was inhibited with 0-10 $\mu \mathrm{g} / \mathrm{ml}$ silkworm extract, recombinant $27 \mathrm{kDa}$ glycoprotein, or recombinant tropomyosin. (B) Silkworm extracts were separated on 12\% SDS-PAGE and IgE-reactive components were probed with patient sera. IgE, immunoglobulin E; M, molecular mass standards; O, no inhibition; G, inhibited by recombinant $27 \mathrm{kDa}$ glycoprotein; T, inhibited by recombinant tropomyosin.

silkworm tropomyosin was $53.3 \%$ (eight of 15 samples), as examined by ELISA of sera from patients with SWP allergy compared with healthy controls (Fig. 2B).

Inhibition of specific IgE binding to total extract. Specific IgE inhibition analysis against total extract of SWP was performed using pooled sera from two patients who exhibited the strongest IgE reactivity to the recombinant SWP tropomyosin. Total extract of SWP inhibited up to $90.2 \%$ of IgE reactivity to total extract of SWP, whereas the recombinant tropomyosin exhibited a maximum $9.7 \%$ inhibition (Fig. 3A). Recombinant 27-kDa hemolymph glycoprotein inhibited IgE reactivity to the extract by up to $11.7 \%$. Inhibition immunoblotting was also performed. However, no specific inhibition of response to the silkworm extract by the recombinant tropomyosin and $27 \mathrm{kDa}$ glycoprotein was observed (Fig. 3B).

Identification of tropomyosin in the SWP extract. Western blotting was performed to identify tropomyosin in the total SWP extract (Fig. 3), since IgE reactivity to total SWP extract was not inhibited by recombinant tropomyosin. Native silkworm tropomyosin, at $\sim 36 \mathrm{kDa}$, was strongly recognized by a monoclonal antibody raised against recombinant German cockroach tropomyosin, indicating that the minimum inhibition of $\operatorname{IgE}$ reactivity by recombinant tropomyosin was not due to a low concentration of native tropomyosin in the extract (Fig. 4).

Correlation of IgE reactivity to silkworm and shrimp/crab tropomyosins. Since shellfish tropomyosins account for $>80 \%$ of IgE reactivity to shellfish in patients, IgE reactivity to shellfish (shrimp and crab) was measured by ImmunoCAP in order to examine its possible cross-reactivity with SWP (Table I). Of the 15 sera tested from patients with silkworm allergy, 11 exhibited IgE reactivity to both shrimp and crab (ImmunoCAP $>0.35 \mathrm{kU}_{\mathrm{A}} / \mathrm{l}$; Table I). Sensitization to $\mathrm{SWP}$ tropomyosin and shrimp or crab extracts did not exhibit a correlation $[\rho=-0.066$ (shrimp, $\mathrm{P}=0.815$ ) and -0.125 (crab, $\mathrm{P}=0.658]$, whereas specific IgE reactivity to shrimp and crab were strongly correlated $(\rho=0.987, P=1.304 \mathrm{e}-011)$. However,
A

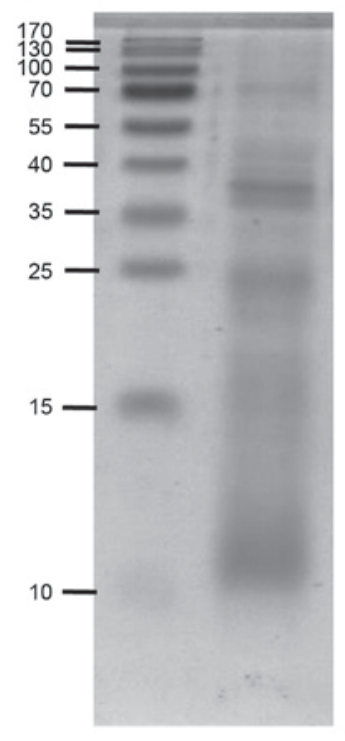

Figure 4. Identification of tropomyosin in the silkworm pupa extract. Silkworm pupa total extract $(10 \mu \mathrm{g})$ (A) was analyzed by $12 \%$ SDS-PAGE and stained with Coomassie Blue, and (B) tropomyosin was detected by western blotting using the monoclonal antibody $2 \mathrm{G} 32$ raised against recombinant German cockroach tropomyosin.

six of eight sera that exhibited positive reactivity to SWP tropomyosin also displayed positive IgE reactivity to shrimp and crab, implying potential cross-reactivity between SWP tropomyosin and shellfish (shrimp/crab) tropomyosin (Table I).

\section{Discussion}

IgEreactivityofrecombinant silkworm tropomyosin.Epidemics of food allergy reflect the regional culture, including eating habits. For example, buckwheat, chestnut, chickpea, bird's nest and royal jelly are frequent causes of food allergy in Asian populations (14). In particular, parvalbumin and tropomyosin proteins are known to be responsible for fish and shellfish 
allergies (15). SWP is an important cause of food allergy in East Asia. In Korea, SWP is commonly consumed after boiling with soybean sauce. Silkworm belongs to the phylum Arthropoda, similar to crustaceans, and the well-established, heat-stable, allergenic nature of tropomyosin in crustaceans was the motivation behind investigating silkworm tropomyosin in the present study. The current study demonstrated that silkworm tropomyosin shared 73.5 to $92.3 \%$ sequence identity with other allergenic tropomyosins, implying possible cross-reactivity. Additionally, recombinant SWP tropomyosin was recognized by serum IgE from $53.3 \%$ of the patients tested with SWP allergy. However, the IgE titer to tropomyosin was not high and its inhibition of IgE reactivity to the total extract of SWP was minimal. Minimum inhibition of $\mathrm{IgE}$ reactivity by recombinant tropomyosin may reflect its low IgE reactivity, as an abundant amount of native tropomyosin was detected in the total SWP extract by western blotting. Non-specific binding or the presence of clinically irrelevant specific IgE binding are common problems in the diagnosis of SWP allergy (5,16-18), as inhibition of the total extract may not reflect clinical significance. In fact, a high prevalence of IgE reaction to SWP is detected in Koreans without clinical symptoms. Therefore, there is an urgent need for component-resolved diagnosis of SWP allergy based on molecular studies.

Potential cross-reactivity with shellfish tropomyosins. The IgE reactivity to shellfish tropomyosin (shrimp and crab), which is known to be responsible for most cases of shellfish allergy, was measured in sera from patients with silkworm allergy by ImmunoCAP, (Table I). Of the 15 sera tested, 11 exhibited $\mathrm{IgE}$ reactivity to shrimp and crab $\left(>0.35 \mathrm{kU}_{\mathrm{A}} / \mathrm{l}\right)$, suggesting possible cross-reactivity between SWP and shellfish tropomyosins. However, none of the patients enrolled were allergic to shrimp and crab. The small amount of tropomyosin in the SWP extract may in part explain the low allergenicity of SWP tropomyosin. For example, cross-reactivity between house dust mite and storage mite tropomyosins (Der f 10 and Tyr p 10) is limited because of its low concentration (19). However, chironomid tropomyosin, Chi k 10, has been demonstrated to be a major allergen, implying a high concentration of tropomyosin in dried dead debris (20). In a recent study, tropomyosin was demonstrated to have a minor role in cross-reactivity among edible insects (21). However, low degree of $\operatorname{IgE}$ reactivity may explain a minor role of tropomyosin in the case of SWP, because it was strongly recognized by monoclonal antibody in the extract used in the current study.

Arginine kinase (Bomb m 1) was identified as a major allergen from SWP (22). However, this result was not reproduced in the present study. IgE reactivity to a 27 kDa-hemolymph glycoprotein and high molecular weight proteins have been demonstrated to be increased following heat treatment (11). Recently, proteins homologous to paramyosin and chitinase have been identified to be allergenic by proteomic analysis (23). In addition, six $\mathrm{IgE}$ reactive components [vitellogenin, chitinase, $30 \mathrm{~K}$ protein homologous to microvitellogenin (Bom m 9), triosephosphate isomerase, heat shock protein, and chymotrypsin inhibitor] from SWP were demonstrated to be recognized by sera from asthma subjects (24). However, IgE reactivity of the purified proteins has not been examined in detail. Therefore, more investigations are needed to characterize allergens and develop improved diagnostics for SWP allergy.

In conclusion, recombinant SWP tropomyosin, sharing strong homology with other allergenic tropomyosins, was produced and demonstrated to exhibit an IgE binding frequency of $53.3 \%$ among patients with SWP allergy. Tropomyosin may contribute significantly to SWP allergy and its importance should not be underestimated because of potential strong cross-reactivity. Large-scale investigations of SWP tropomyosin and its potential allergenic cross-reactivity will be required in the future for further characterization in order to develop more efficient diagnostic tests for SWP allergy.

\section{Acknowledgements}

This research was supported by a grant of the Korea Healthcare Technology R\&D Project through the Korean Health Industry Development Institute, Funded by the Ministry of Health, Welfare \& Family Affairs, Republic of Korea (grant no. HI14C1324).

\section{References}

1. DeFoliart GR: Insects as food: Why the Western attitude is important. Annu Rev Entomol 44: 21-50, 1999.

2. Pemberton RW: Insect and other arthropods used as drugs in Korean traditional medicine. J Ethnopharmacol 65: 207-216, 1999.

3. Ramos-Elorduy J: Insects: A sustainable source of food? Ecol Food Nutr 36: 247-276, 1997.

4. Ji KM, Zhan ZK, Chen JJ and Liu ZG: Anaphylactic shock caused by silkworm pupa consumption in China. Allergy 63: 1407-1408, 2008.

5. Kim SH, Kang HR, Kim KM, Kim TB, Kim SS, Chang YS, Kim CW, Bahn JW, Kim YK, Cho SH, et al: The sensitization rates of food allergens in a Korean population: A multi-center study. J Asthma Allergy Clin Immunol 23: 502-514, 2003.

6. Komaze Y, Sakata M, Azuma T, Tanaka A and Nakagawa T: IgE antibodies against midge and moth found in Japanese asthmatic subjects and comparison of allergenicity between these insects. Allergy 52: 75-81, 1997.

7. Baldo BA and Panzani RC: Detection of $\operatorname{IgE}$ antibodies to a wide range of insect species in subjects with suspected inhalant allergies to insects. Int Arch Allergy Appl Immunol 85: 278-287, 1988.

8. Jeong KY, Hong CS and Yong TS: Allergenic tropomyosins and their cross-reactivities. Protein Pept Lett 13: 835-845, 2006.

9. Altschul SF, Gish W, Miller W, Myers EW and Lipman DJ: Basic local alignment search tool. J Mol Bio 215: 403-410, 1990.

10. Larkin MA, Blackshields G, Brown NP, Chenna R, McGettigan PA, McWilliam H, Valentin F, Wallace IM, Wilm A, Lopez R, et al: Clustal W and Clustal X version 2.0. Bioinformatics 23: 2947-2948, 2007.

11. Jeong KY, Son M, Lee JY, Park KH, Lee JH and Park JW: Allergenic characterization of 27-kDa glycoprotein, a novel heat stable allergen, from the pupa of silkworm, Bombyx mori. J Korean Med Sci 31: 18-24, 2016.

12. Jeong KY, Lee J, Lee IY, Ree HI, Hong CS and Yong TS: Allergenicity of recombinant Bla g 7, German cockroach tropomyosin. Allergy 58: 1059-1063, 2003.

13. Jeong KY: Characterization of allergenic properties of German cockroach tropomyosin using recombinant proteins, Yonsei University College of Medicine, 2004.

14. Lee BW, Shek LP, Gerez IF, Soh SE and Van Bever HP: Food allergy-lessons from Asia. World Allergy Organ J 1: 129-133, 2008.

15. Leung NY, Wai CY, Chu S, Wang J, Kenny TP, Chu KH and Leung PS: Current immunological and molecular biological perspectives on seafood allergy: A comprehensive review. Clin Rev Allergy Immunol 46: 180-197, 2014.

16. Lee MH, Kim YY and Kang SY: Two cases of pupa allergy. Allergy 2: 23-25, 1982. 
17. Kim S, Paik SW and Kang SY: A case of anaphylaxis after ingestion of pupae. Allergy 3: 175-178, 1983.

18. Koh YI, Choi IS, Chung SU and Cho S: Clinical features of adult patients with anaphylaxis associated with food in Gwangju and Chonnam area. J Asthma Allergy Clin Immunol 24: 217-223, 2004.

19. Jeong KY, Lee H, Lee JS, Lee J, Lee IY, Ree HI, Hong CS and Yong TS: Molecular cloning and the allergenic characterization of tropomyosin from Tyrophagus putrescentiae. Protein Pept Lett 14: 431-436, 2007.

20. Jeong KY, Yum HY, Lee IY, Ree HI, Hong CS, Kim DS and Yong TS: Molecular cloning and characterization of tropomyosin, a major allergen of Chironomus kiiensis, a dominant species of nonbiting midges in Korea. Clin Diagn Lab Immunol 11: $320-324,2004$.
21. Srinroch C, Srisomsap C, Chokchaichamnankit D, Punyarit P and Phiriyangkul P: Identification of novel allergen in edible insect, Gryllus bimaculatus and its cross-reactivity with Macrobrachium spp. Allergens. Food Chem 184: 160-166, 2015.

22. Liu Z, Xia L, Wu Y, Xia Q, Chen J and Roux KH: Identification and characterization of an arginine kinase as a major allergen from silkworm (Bombyx mori) larvae. Int Arch Allergy Immunol 150: 8-14, 2009.

23. Zhao X, Li L, Kuang Z, Luo G and Li B: Proteomic and immunological identification of two new allergens from silkworm (Bombyx mori L.) pupae. Cent Eur J Immunol 40: 30-34, 2015.

24. Zuo J, Lei M, Yang R and Liu Z: Bom $m 9$ from Bombyx mori is a novel protein related to asthma. Microbiol Immunol 59: 410-418, 2015. 